\title{
Hacia una formalización de los procesos implicados en la comprensión musical
}

\section{Emmanuel Bigand}

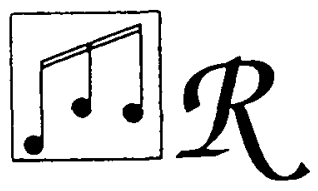

El principal problema que se plantea en la comparación músicallenguaje estriba en la naturaleza de los procesos implicados en la percepción y comprensión de las producciones musicales. En este artículo se plantean ciertas precauciones a la bora de trasponer sin más los modelos de análisis formales del lenguaje a la música, abogando por la teoría generativa de Lerdabl y Jackendoff y analizando sus implicaciones para la formación musical.

La música es una forma de expresión simbólica compleja y misteriosa que permite a los seres humanos reflejar de forma sonora cómo sienten y se representan el mundo que les rodea. Es pues fundamental como una forma elaborada de comunicación humana no verbal basada en una doble competencia: la posibilidad de incluir en una serie de signos acústicos elementos portadores de significado y la posibilidad de encontrar dichos elementos para comprender el significado implícito en esta forma. De ello surge la posibilidad de que los investigadores se planteen dos interrogantes.

En primer lugar nos podemos preguntar por la naturaleza de los significados musicales, teniendo como planteamiento fundamental la comprensión de cuáles de estos significados son específicos e irreductibles a los propios de otras formas de comunicación, en especial el lenguaje (Francès, 1958; Imberty, $1979,1981)$. Pero también podemos reflexionar sobre las posibilidades de la música como forma de expresión: ¿Cómo y en virtud de qué principios una serie de signos acústicos consiguen establecer significados referidos a algo que no sean ellos mismos? El planteamiento teórico en este caso es muy diferente y podemos suponer que la música se basa, al menos a un cierto nivel, en los mismos principios que los que rigen cualquier otra forma de comunicación simbólica, incluido el lenguaje (Ruwet, 1972; Clarke, 1989)..

Es en este planteamiento en el que podemos inscribir la analogía entre música y lenguaje; analogía muy antigua, de la que deriva la noción de 
comprensión musical. Pero, ¿se trata sólo de una simple analogía? Habitualmente la relación entre los dos campos se plantea analizando los aspectos más externos de las actividades musicales y lingüísticas, sin embargo esto no es lo esencial: la música y el lenguaje son dos formas de comunicación que implican la existencia de una competencia específicamente humana para comunicar mediante formas sonoras. Las actividades que derivan de esta competencia posiblemente tienen un cierto número de puntos en común, aunque, evidentemente, es probable que esta competencia se divida en competencias específicamente musicales y lingüísticas. Un progreso importante en el estudio de los comportamientos musicales y linguísticos sería concretar esta especificidad, la cual beneficiaria tanto a las ciencias de la música (Lerdahl, 1987) como a las investigaciones psicológicas y pedagógicas.

Si se nos sigue en este planteamiento, podremos analizar brevemente las características fundamentales de los comportamiento linguiísticos ofrecidos por las investigaciones psico-lingüísticas para analizarlas después en la música.

\section{TEORIA DEL LENGUAJE - TEORIA DEL LENGUAJE MUSICAL}

La primera característica es esencial: se refiere al aspecto creador del lenguaje humano. Desde el punto de vista del hablante, la creación se traduce en la posibilidad de producir frases muy variadas, que expresan pensamientos nuevos y diferenciados. Desde el punto de vista del oyente la creación se traduce en la posibilidad de asignar un sentido a frases que antes no se habían oído. Este aspecto innovador permite la comunicación de informaciones sobre el mundo constantemente renovadas, contribuyendo así al desarrollo del conocimiento. Esto hace que la creatividad del lenguaje sea una cuestión esencial, en la que cualquier teoría linguística o psicolinguística aspira a poder hacer algún descubrimiento relevante. Es un argumento importante, que llevó al lingüista N. Chomsky (1969), y a un importante número de investigadores, a rechazar las explicaciones de los comportamientos lingüísticos que se basaban en las teorías del aprendizaje (Massino-Pattieli-Palmerini, 1979). El mero hecho de poder crear y comprender una infinidad de frases nuevas demuestra, efectivamente, que el lenguaje no se puede reducir a un sistema de hábitos activado por estímulos exteriores y basado en la imitación y el condicionamiento.

En segundo lugar, esta capacidad de creación se puede formalizar con ayuda de un número limitado de reglas que constituyen una gramática generativa transformacional. Según la hipótesis fundamental de la teoría de Chomsky, que sigue de actualidad a pesar de las modificaciones que ha sufrido, esta gramática constituiría un modelo de la competencia linguística humana. Comprendería un primer grupo de reglas para describir la generación de la "frase núcleo" y un segundo grupo que describiría las diferentes transformaciones aplicadas a estas frases núcleo. Comprender una frase implicaría, por tanto, asimilar las diferentes transformaciones que permiten llegar a la frase entendida. De ello surge una distinción fundamental entre la estructura superficial de una frase, que se define por la sucesión sintagmática, y su estructura profunda, que está formada "por la sucesión de bases constituidas por relaciones funcionales del tipo de «sujeto de», "predicado de" y "verbo principal de», y "por las operaciones que se pueden efectuar para llegar a 
la frase de superficie» (Moscato y Wittwer, 1981, p. 51). Por tanto la interpretación de una frase se debería hacer a nivel de su estructura profunda. Por eso, para que haya comunicación verbal es necesario, incluso aunque actualmente parezca que pueden intervenir otros factores, que el hablante y el oyente tengan una representación cognitiva de esta gramática.

La distinción de las dos estructuras nos lleva a la tercera característica: el sentido en el lenguaje no se reduciría sólo a la presencia de palabras con significado a nivel de estructura superficial de la frase. El significado también es el resultado del sistema de relaciones existentes entre dichas palabras. Consideremos, por ejemplo, la siguiente frase inglesa: «That the was happy was evident from the way he smiled». Para comprenderla es necesario identificar el sintagma nominal, después el sintagma verbal, a continuación descubrir en éste dónde se encuentra el verbo y el complemento, el conjunto de estas relaciones se puede representar en forma de árbol (Fig. 1). La abstracción de esta red de relaciones nos permite comprender que el que «él era feliz era evidente por su forma de sonreír». "Comprender una frase consiste, por lo tanto, en establece cognitivamente la red de relaciones, teóricamente representables en forma de gráfico, a la que esta frase da acceso" (Noizet, 1980 , p. 131). Dos frases con una misma estructura superficial pero de estructura profunda diferente no tienen el mismo significado $^{1}$ y viceversa.

Figura 1

Red de relaciones en una frase

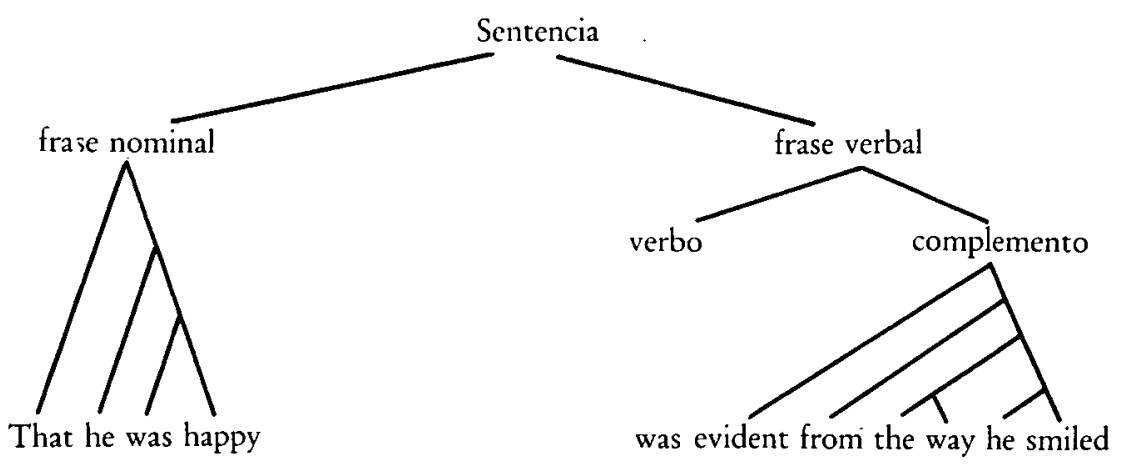

Esta tercera característica demuestra que las cosas sólo adquieren sentido lógico en la medida en que se insertan en una red de relaciones. O dicho de otra forma, los significados comunicados por el lenguaje no se refieren tanto a entidades inertes y aisladas como a las relaciones existentes entre ellas. Lo ilustraremos con un ejemplo tomado de Molino y utilizado más tarde por Nattiez (1987, p. 32). Consideremos los enunciados de la forma "A es a B lo que $\mathrm{X}$ es a $\mathrm{Y}$ ", si atribuimos palabras al azar a cada una de las letras, podremos obtener la frase siguiente «El cepillo de dientes es a Dios lo que Verdi es a los italianos». A pesar de su aparentemente incongruencia sería difícil afirmar que esta frase no tiene ningún significado. El sistema de las relaciones existentes entre las palabras tiene significado en sí mismo. 
Estas tres características se pueden resumir esquemáticamente de la forma siguiente; en el lenguaje el significado está asegurado, de principio, por la red de relaciones que une la sucesión de signos acústicos (característica 3). La creación y comprensión de esta red está asegurada por un conjunto de reglas que definen la competencia linguística necesaria para poderse comunicar mediante el lenguaje (característica 2). Estas reglas tienen un número finito y permiten engendrar una infinidad de frases comprensibles, lo que evidencia el aspecto creador del lenguaje (característica 1 ).

$¿$ Hasta qué punto se pueden entrever estas mismas características en el caso de la música y qué consecuencias podría tener esto para el estudio de los procesos de comprensión musical?

Lo primero no parece plantear problemas. Efectivamente, podemos afirmar que la característica fundamental de la música es su aspecto creador: como en el lenguaje, la creación se encuentra en la posibilidad de engendrar una infinidad de obras, cuya especificidad nos remite a la naturaleza de los sentimientos y pensamientos que el compositor ha intentado expresar. En el caso de la música escrita, el acto creativo se sitúa también a nivel del intérprete. El problema se plantea en el caso del oyente. Cuando se hace referencia al oyente habitualmente se prefiere hablar de recepción más que de verdadera creación, subrayando su carácter pasivo. Así, el oyente se limitaría a registrar una serie de episodios con unas cualidades sonoras más o menos brillantes que le transportarían a un mundo subjetivo de ensueños diversos y variados. Aunque este planteamiento responde en gran medida a la realidad ${ }^{2}$, debemos reconocer que no responde a la totalidad de las situaciones de audición musical: en muchos casos los oyentes desarrollan una audición activa en la que tratan de seguir el discurso musical, de reconstruir mentalmente su unidad y descubrir la originalidad de la obra.

Pero incluso en este caso, la actividad desplegada por el oyente, por su naturaleza, podría parecer diferente del acto creador implicado en la comprensión del lenguaje. En efecto, parece que el oyente se limita a los aspectos fisionómicos de la materia sonora y no está capacitado para captar el proceso de composición subyacente seguido por el compositor para crear la obra de una determinada forma y dotarla de una unidad orgánica original. Por ello, el concepto de obra musical sólo tendría realidad psicológica después de un cierto número de audiciones durante las cuales nuestra memoria establecería las conexiones asociativas entre las diferentes secciones que la forman, basándose únicamente en sus relaciones de contigüidad temporal. El conocimiento de la obra sería entonces puramente descriptivo, y su significado implicaría numerosas repeticiones durante las cuales se iría asociando a la obra, minuciosamente, un sentido subjetivo. Según este planteamiento, la percepción y comprensión de la obra musical sólo tendría realidad psicológica después de un cierto número de audiciones durante las cuales nuestra memoria establecería las conexiones asociativas entre las diferentes secciones que la forman, basándose únicamente en sus relaciones de contigüidad temporal. El conocimiento de la obra sería entonces puramente descriptivo, y su significado implicaría numerosas repeticiones durante las cuales se iría asociando a la obra, minuciosamente, un sentido subjetivo. Según este planteamiento, la percepción y comprensión de la obra musical serían radicalmente diferentes de las del lenguaje. 
Es cierto que la comprensión de una obra mejora con el número de audiciones, pero ello no nos debe ocultar otro aspecto esencial: todo oyente, incluso cuando se enfrenta a una obra desconocida, llega a abstraer una cantidad considerable de información que supera ampliamente el campo de las características fisionómicas/superficiales de la materia sonora y que se relaciona directamente con los procesos de composición. Puede decir, por ejemplo, si la pieza pertenece al repertorio de la música occidental, si se trata de música clásica, romántica, contemporánea, de jazz, de rock o ligera. En muchos casos podría precisar la naturaleza de la forma (tema variado, sonata, fuga, etc.) y tener una idea concreta de los significados comunicados por la obra. Es fácil comprobar que una sola audición del "pasatiempo musical» de Mozart basta a dos sujetos no músicos para comprender que la intención del compositor no es la misma que en otras obras comparables por sus aspectos superficiales. Varias experiencias llevadas a cabo por M. L. Serafine (1989) demuestran, además, que desde la primera audición se realiza la abstracción de la estructura de un breve fragmento musical, aunque los aspectos relativos a las superficies musicales se codifican posteriormente (Op. cit., p. 414).

Por tanto, el oyente tiene una competencia musical que le basta para asimilar desde la primera audición diferentes aspectos de los procesos creativos engendrados por la composición; manifiesta así una actividad de creación (o de recreación) comparable a la que interviene en la comprensión del lenguaje. Si no fuera así sería difícil explicar el extraordinario placer de descubrir músicas «de oído» y la posibilidad de asimilar la especificidad de una obra. Sería totalmente ilusorio intentar descubrir algo de la intención composicional e imposible considerar la música como una forma elaborada de comunicación humana no verbal, $y$, por tanto, hablar de comprensión en música.

Pero ¿̇hasta qué punto pueden ser semejantes los procesos que rigen la creación musical (composición y percepción-comprensión) a los del lenguaje?

Ya hemos señalado con anterioridad que el aspecto creador en el lenguaje se traducía en la posibilidad de producir y comprender una infinidad de secuencias verbales con ayuda de un número finito de reglas de producción. Nos puede parecer algo sorprendente a priori descubrir algo semejante en la música, en la que una cierta imagen social se complace en representar la creación artística como algo liberado de todo tipo de ataduras, y al compositor, como un Dios todopoderoso, creando un mundo sonoro de la nada, siguiendo únicamente las inclinaciones de su humor y su fantasía. Esta ingenua concepción está tal falta de base que hace necesarias algunas precisiones sobre teoría de la música: nuestra música occidental obedece a reglas armónicas y de contrapunto muy estrictas, con las que los compositores, incluso los más revolucionarios, crean sus obras. También debemos reconocer con B. De Schloezer (1974, p. 119-120) que «el hombre sólo puede crear según un determinado plan»; su obra "está condicionada en todas sus etapas, por determinadas reglas que fijan su desarrollo, manteniéndola dentro de unos estrechos límites". Incluso la creación musical contemporánea, que al parecer había abolido todo el sistema, reivindica esta noción de reglas (Boulez, p. 1985 , p. 273 y siguientes) y su importancia para la creación se manifiesta concretamente en el desarrollo de la Composición asistida por ordenador (IRCAM, 1989). 
Pero el parecido más llamativo entre música y lenguaje no es la existencia de reglas, sino el hecho de que estas reglas sean de un número finito. En este aspecto nos vamos a detener brevemente, pues su repercusión sobre las investigaciones musicales y en pedagogía podrían ser importantes.

Recordemos que hay dos grandes corrientes en el análisis musical. En una, los análisis se centran sobre todo «en la identificación de temas, en su enumeración, su organización retórico musical incluida dentro de la música tonal». El conjunto de este sistema de análisis «tenía en común el desembocar en una localización, identificación y clasificación de los elementos musicales, evaluados estadísticamente tomando como base la frecuencia de su aparición en los procesos: todo intento de estilística pasaba por este tipo de evaluación». Por el contrario, en el otro tipo de análisis se intentaba descubrir el «sistema de reglas que ha engendrado la gramática tonal y le ha otorgado un estatus a través de la pluralidad de estilos" (C. Deliège, 1984, p. 41 y siguientes).

El musicólogo H. Shenker, nacido en 1867 y muerto en 1935, es el líder de ete segundo tipo de enfoque: aparece como el precursor del lingüista $\mathrm{N}$. Chosmky. Su importancia estriba en «haber descubierto la forma de las estructuras subyacentes a todo el proceso tonal, tanto en el plano de la armonía como en el del contrapunto, y haber mostrado la forma de inclusión de todos los procesos secundarios, modulantes y no modulantes, en la estructura de base» (Deliège, op. cit., p. 52). Debemos diferenciar tres estructuras: «La estructura generadora inicial» (Ursatz), la «base generadora mediana (Mittelgrund) y, por último, la base generadora de superficie (Vordergrund). La estructura generadora inicial (“Ursatz») es estrictamente diatónica y establece la tonalidad principal del fragmento (Fig. 2). Podemos decir, a título de ilustración, que esta estructura es a la música tonal lo que la estructura sintagma nominal/sintagma verbal es al lenguaje. Enseguida se enriquece dentro de la estructura generadora mediana con prolongaciones. Ello hace aparecer los grados de la progresión intermedia entre tónica y dominante así como las modulaciones de la pieza, subordinadas a la tonalidad principal. Por último, la estructura generadora de superficie nos aproxima aún más a la partitura real, ante todo con la mención de todas las progresiones armónicas locales. Todos los procesos terminales (superficies musicales), derivan así de una misma estructura formal de base (Ursatz) según un número finito de principios. Por tanto, en música, como en el lenguaje, se puede definir una gramática generativo-transformacional. Esta posibilidad se concreta en la realización de programas informáticos que permiten crear, con ayuda de un número finito de algoritmos, una infinidad de frases musicales tonales correctas y conformadas a un estilo de referencia (Lidov, 1975; Sundberg Lindblom, 1976; Baroni, 1989).

La forma de combinar las reglas de esta gramática, «de explotar los recursos de un lenguaje» es lo que va a definir el estilo de la composición (C. Rosen, 1978, p. 185) e «informar» la materia sonora de la intención del compositor. Una parte esencial del significado musical residiría pues en la asimilación de los procedimientos de derivación que engendran los procesos terminales ${ }^{3}$.

Veamos ahora cuál es la tercera característica común a la música y el lenguaje. Ya dijimos anteriormente que «la interpretación de una frase (del lenguaje) lleva a buscar su significado en su estructura profunda, haciendo 
Figura 2

Ejemplo de estructuras shenkerianas: arriba se encuentra el Ursatz, en el centro el Mittelgrund y abajo el Vordergrund.
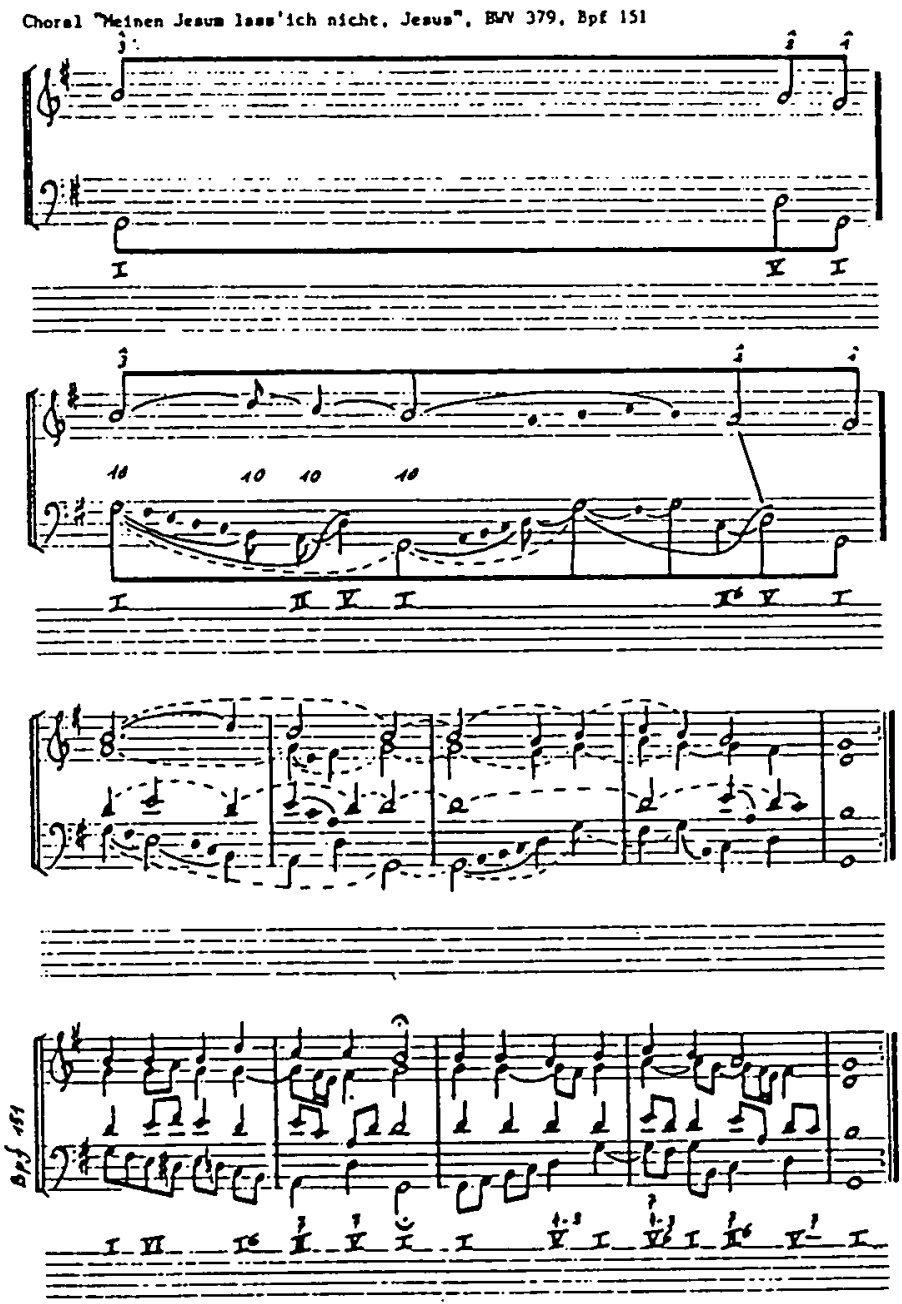

(...) las transformaciones que permiten el paso de las series de base a la estructura superficial» (M. Moscato et al., Op. cit., p. 52). En adelante, podremos decir que «el secreto del equilibrio, en música, reside en la consciencia permanente de los niveles de transformaciones y del movimiento de la estructura superficial hacia la estructura generadora inicial, o del movimiento inverso" (Schenker, 1935, citado por Deliège, Op. cit., p. 59). Como en el lenguaje, en música los diferentes niveles de transformaciones se pueden representar por una red jerárquica de relaciones que definirá lo que llamamos la estructura subyacente de una pieza musical (Fig. 3). En este árbol los elementos enraizados más profundamente son los más ornamentales, mientras que los elementos que sólo comprenden un enlace son los más importantes estructuralmente. 
Figura 3

Red jerárquica de las relaciones subyacentes a un fragmento musical (según Lerdabl y Jackendoff, 1983)
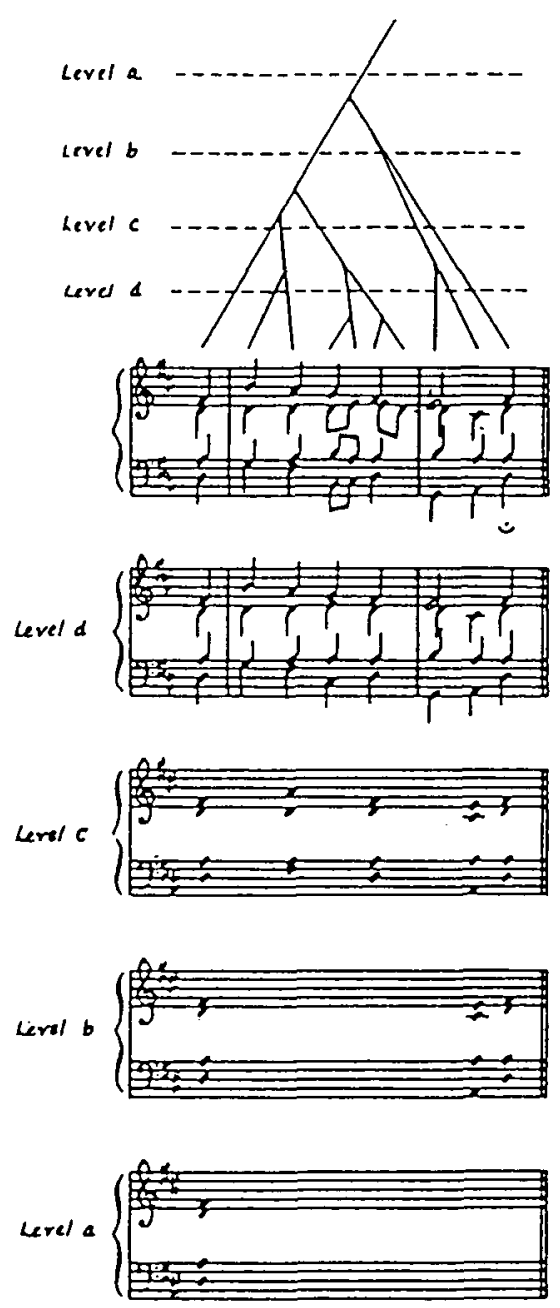

Por tanto, podemos plantear como hipótesis de trabajo la siguiente definición de la comprensión musical: «Comprender una pieza musical consiste en establecer cognitivamente la red de relaciones jerárquicas, teóricamente representable en forma de gráfico, a lo que dicha pieza da acceso». Más adelante precisaremos en qué sentido esta definición supera ampliamente el marco de una concepción puramente intelectualista de la percepción y la comprensión musical y explica una parte importante de los significados en música.

Nos parece que sería interesante esbozar cuáles pueden ser las consecuencias para la investigación en psicología de la música de esta clara cercanía entre música y lenguaje. Ante todo vamos a referirnos a los aspectos relacionados con el estudio de la percepción y la comprensión musical, pero, como conclusión, nos referiremos también a las posibilidades que esa proximidad ofrece a la pedagogía de la música. 
GRAMATICA COMPOSICIONAL Y MODELO DE LA AUDICION

Del anterior planteamiento se desprende que la comprensión musical implica que el oyente posee una representación cognitiva del sistema de reglas que han determinado la composición, sistema que podemos designar como gramática composicional (Lerdhl, 1987). Aunque es evidente que un modelo de esta gramática no puede ser en ningún caso modelo de audición, ya que las reglas que intervienen en la generación de los procesos musicales no dan cuenta de las reglas heurísticas que intervienen en la decodificación de las piezas musicales: en ella intervienen necesariamente un número importante de trabas específicas a la situación de audición. Formalizar las diferentes trabas podría ser el objetivo central de la psicología de la percepción musical. La investigación contribuiría así al dominio de las ciencias de la música, ya que el grado de comprensión musical se podría definir por la mayor o menor adecuación entre las gramáticas composicionales y el modelo de audición (Lerdahl, 1987), pero también sería interesante para la psicología, ya que la definición de este modelo nos permitiría centrarnos en los procesos de decodificación de las formas simbólicas que se desarrollan en el tiempo.

¿Cómo podremos llegar a dicha definición? Revisando las investigaciones experimentales se descubre que hay un número importante de factores a tener en cuenta. Su diversidad nos podría desanimar ante la imposibilidad de llegar a un modelo unificado de la comprensión musical. Sin embargo es importante ver que los diferentes estudios descubren tres categorías principales de factores: la primera está relacionada con el conocimiento implícito de las jerarquías intratonalidades e inter-tonalidad establecidas por el sistema tonal. Numerosos estudios confirman que el oyente occidental tiene una representación mental de dichas jerarquías (ver, por ejemplo, Krumhansl y Kessler, 1982). La segunda se relaciona con los principios de organización perceptiva, totalmente independientes de las jerarquías establecidas por el sistema tonal, y que dependen esencialmente de las características de nuestros sistemas sensoriales de organización del campo perceptivo. Estos principios describen sobre todo la forma en que una superficie musical se puede organizar perceptivamente en forma (Gestalt) de pequeños fragmentos temporales y, a partir de ello, desprenderse de estructuras de periodicidades métricas. La última se refiere a la forma en que la información descubierta localmente dentro de los grupos, puede combinarse a niveles superiores de organización. Nos descubre las trabas cognitivas generales implicadas en la asimilación de las relaciones existentes entre elementos que se prolongan en el tiempo ${ }^{4}$. Estas tres categorías de factores orientan y determinan la percepción y la comprensión musical. Sin embargo su enumeración no constituye un modelo de la audición: Hoy por hoy parece esencial formalizar cómo interactúa el conjunto, pasando de la percepción de una superficie musical a la comprensión de la red de relaciones subyacente.

\section{LA TEORIA GENERATIVA DE LA MUSICA TONAL DE LERDAHL Y JACKENDOFF (1983)}

Esta teoría constituye una importante aportación teórica. Los autores, un compositor y un lingüista, desarrollan un modelo cuya finalidad global 
es ofrecer una descripción formal de las estructuras inferidas por el oyente "experimentado" cuando oye y comprende una obra más allá de los datos puramente acústicos. Comprende cuatro componentes (Fig. 4): «las estructuras de agrupamiento» describen cómo en la audición la pieza se organiza en una segmentación jerárquica; "las estructuras métricas" formalizan las relaciones existentes, en numerosos niveles jerárquicos, entre los pasajes de la pieza y la alternancia regular de tiempos fuertes y débiles; "las reducciones de las tramas temporales» atribuyen a los elementos de la pieza una jerarquía de importancia estructural; por último, "la reducción prolongacional" describe las jerarquías de tensiones y distensiones armónicas y melódicas inferidas por el oyente (Lerdahl et al., p. 9-10). El modelo define para cada uno de estos componentes reglas de correcta formación, que explicitan las diferentes formas de estructura posibles, y reglas preferenciales que determinan qué estructura será inferida en un determinado caso.

\section{Figura 4}

Representación de los cuatro componentes del modelo de Lerdabl y Jackendoff

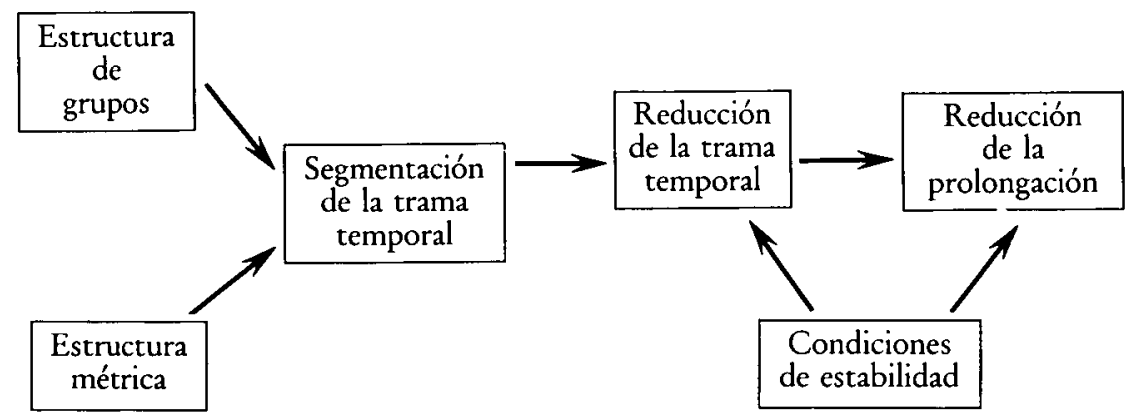

El primer componente comprende doce reglas que determinan la estructura de agrupamiento percibida en un determinado tipo de configuración musical. Por ejemplo, entre las dos configuraciones teóricamente posibles de la figura 5, el modelo permite predecir, en virtud de las reglas 2 y 3 , que el oyente percibirá preferentemente la estructura del grupo b. Estas reglas muestran el efecto de la diferencia de registro, dinámica, duración, articulación y paralelismo en la formación de grupos. Su pertinencia perceptual ha sido confirmada en gran parte por I. Deliège $(1985,1987)$. Este primer componente permite, por tanto, formalizar cómo todos los pequeños detalles que existen a nivel de superficies musicales intervienen en la segmentación. La observación del modelo permitirá comprender hasta qué punto esos detalles pueden ser fundamentales para la comprensión de la obra.

El segundo componente describe el tipo de estructura métrica con la que el oyente relaciona todos los episodios de la pieza. Las reglas de correcta formación definen las configuraciones posibles (Fig. 6); las reglas 
Ficiura 5

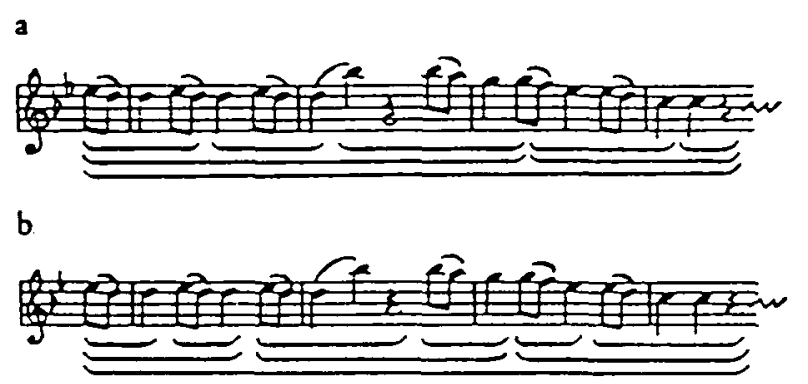

Dos estructuras de agrupamiento posibles (según Lerdabl et al.)

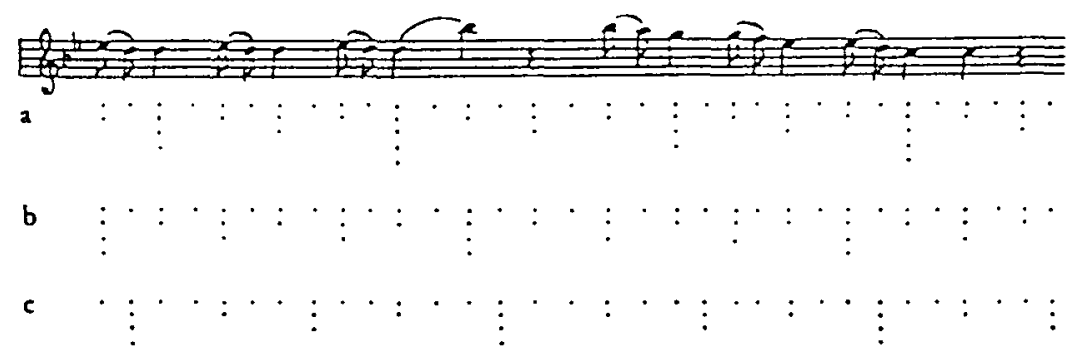

Tres estructuras métricas posibles (según Lerdabl et al.)

preferenciales permiten predecir la estructura realmente inferida por el oyente. En el presente caso la regla número 5, que estipula que el tiempo fuerte debe coincidir preferentemente con un episodio sonoro de una duración relativamente larga, permite seleccionar la configuración a.

Estos dos primeros componentes caracterizan a un número importante de estructuras musicales, como por ejemplo la síncopa y el contratiempo. Además, describen la segmentación perceptual de la pieza en lapsos de tiempo (time span) de diferente envergadura temporal. Aunque son indispensables para el modelo, sin embargo no implican más que las intuiciones auditivas relativas a los aspectos superficiales de la música, y no permiten formalizar todo aquello que se relaciona con el lenguaje musical, sobre todo con las jerarquías de altura (intra e inter tonalidades): ¿por qué, por ejemplo, un oyente percibe una diferencia entre la versión original de la Fuga a 6 voces de la Ofrenda musical de J. S. Bach y la transcripción. de A. Webern? ¿Qué hace para percibir la relación entre un tema y sus variaciones? Los siguientes componentes harán referencia a estos aspectos. 
El tercer componente se basa en la hipótesis de la reducción de las tramas temporales, según la cual «el oyente organiza todos los episodios de la pieza en una estructura coherente única, de forma que cada uno de los episodios se perciba dentro de una jerarquía de importancia relativa y que los sonidos menos importantes estructuralmente, no se perciban como simples inserciones, sino que tengan una relación específica con aquellos elementos cercanos más importantes" (Op. cit., p. 108). Se trata, pues, de formalizar los principios que rigen esta jerarquización, y la idea principal es que la importancia jerárquica de un episodio depende de la estructura métrica y de agrupamiento en la que aparece. Hay dos razones para ello. En primer lugar la importancia de un episodio no sólo depende de su carga total, sino también de su pregnancia perceptual $^{5}$. Además, esta importancia depende de los episodios con los que se le compara, y, por tanto, del grupo de notas al que se va a referir la comparación. Así, «los componentes de agrupamiento y de métrica tienen una doble función en la construcción de las reducciones: segmentan la música en campos rítmicos y, dentro de estos campos, añaden criterios a las jerarquías de altura, más allá de la determinación de la importancia estructural de los episodios (op. cit., p. 119). «Dentro de estas tramas (...) los episodios menos estables son eliminados por reducción en cada nivel, de forma recursiva, hasta que sólo queda un único episodio para el conjunto de la pieza» (F. Lerdahl, p. 112, 1989, ver Fig. 3).

Entre las distintas reglas preferenciales que definen este tercer componente, citaremos, por ejemplo, la regla número 1, que predice que entre las posibles elecciones del elemento dominante en un lapso de tiempo $T$, el oyente percibirá preferentemente lo que tiene una posición métrica fuerte. La regla número 2 hace referencia a la influencia de la consonancia relativa del episodio, y de su peso tonal en relación con la tónica local, y la regla número 3 se refiere a la influencia del contorno musical. Otras reglas formalizan la fijación cadencial: ésta depende de la estructura del grupo en el que dos episodios pueden hacer emerger una cadencia. Por ejemplo, la presencia de acordes de quinto y primer grado no basta para definir un caso de cadencia (ver las ocho primeras medidas del preludio en La mayor de Chopin): ésta depende esencialmente de la segmentación de la superficie musical llevada a cabo por el oyente.

Este conjunto de reglas permite predecir cuál es la estructura jerárquica inferida dentro de cada grupo, y formaliza la interacción entre las estructuras descubicrtas a nivel superficial (grupo y métrica) y las jerarquías de altura establecidas por el sistema tonal. Sin embargo, este componente describe únicamente intuiciones limitadas al marco de diferentes lapsos de tiempo. Se trata de una reducción estática que aisla los episodios importantes, pero no traduce las intuiciones del oyente sobre la progresión de la música más allá de la frontera de los grupos.

Este será el objetivo del último componente. Se basa en la doble hipótesis de la reducción prolongacional, que estipula, por una parte, que la pieza se organiza para la audición en una jerarquía de tensiones y distensiones musicales en la que «cada región representa una tensión y distensión desde su comienzo hasta el final, las tensiones y distensiones internas de cada región representan etapas subordinadas, y no se montan unas sobre otras en la progresión total» (hipótesis 1). Por otra parte, la elección de los episodios que definen la región 
prolongacional está muy influida por la importancia relativa de los episodios en la reducción de las tramas temporales" (hipótesis 2) (ver p. 221).

Las reglas de este último componente son la aportación más importante de la teoría por tres razones. En primer lugar dan cuenta de un fenómeno esencial en la percepción y comprensión musical, sobre el que han insistido numerosos psicólogos y musicólogos: el de los esquemas de tensión y distensión (Francès, 1958; Meyer, 1973; Francès y Bruchon-Schweitzer, 1982; Imberty, 1979, 1981). Además formaliza las repercusiones que tienen para el oyente las diferencias de importancia estructural aisladas a nivel del tercer componente: los episodios estructualmente importantes son episodios estables, sobre los que se puede detener momentáneamente el discurso musical, mientras que los ornamentales son inestables y crean tensiones musicales que exigen solución. Estas diferencias de importancia son jerárquicas, lo que implica, y en ello consiste la primera aportación original del modelo, que los esquemas de tensión y distensión correspondientes se organizan también jerárquicamente. La noción de comprensión musical, tal como la hemos definido anteriormente, toma todo su significado psicológico: hay todas las razones para pensar que es en la forma de engendrar esta jerarquía de tensiones y distensiones, en la forma específica en que cada compositor desarrolla en el tiempo la materia sonora, donde se inscribe su intención composicional, y así surge una parte esencial de los significados comunicados por la música. Una confirmación de este fenómeno la podemos encontrar en el experimento realizado por Imberty $(1979,1981)$ sobre piezas de Brahms (Intermezzo) y Debussy (La puerta del vino). Finalmente, y ésta es la segunda aportación original, la interdependencia entre la reducción y las tramas temporales y la reducción prolongacional, subraya hasta qué punto «los patrones de tensión y distensión percibidos dependen fundamentalmente de la jerarquía de episodios definida por el metro y el agrupamiento" (ver p. 188). Dicho de otra forma, el modelo llega a formalizar la forma en que el conjunto de los factores relativos a los aspectos fisionómicos de la materia sonora y a las jerarquías de altura específicas del sistema tonal interactúan para determinar la red jerárquica de tensiones y distensiones específica de la obra que se está oyendo. Explicita también hasta qué punto toda la serie de pequeños detalles superficiales, tan importantes para el intérprete, pueden influir en la comprensión musical.

\section{REALIDAD COGNITIVA DE LA RED DE RELACIONES JERARQUICAS EN UNA FRASE MUSICAL}

Varios experimentos confirman la realidad psicológica de esta red de relaciones jerárquicas (Palmer y Krumhansl, 1987a y b; Serafine, 1989; Bigand, 1989). Para terminar, nos gustaría ofrecer una breve revisión de un estudio realizado siguiendo este planteamiento (para más detalles sobre el procedimiento, ver Bigand, 1989, exp. 6, y 1990). En primer lugar se define una primera familia de cuatro melodías (a1, b1, c1, d1). Sus estructuras superficiales son distintas, pero tienen la misma estructura subyacente, definida según las reglas del modelo de Lerdahl et al. (Fig. 7). Para cada una de estas melodías se escribe una nueva melodía que tiene exactamente los mismos ritmos y los mismos contornos melódicos, pero que se desarrolla según otra estructura subyacente. Así se obriene una segunda familia de cuatro melodías: a2, b2, c2, d2. 


\section{4}

Figura 7

Dos familias de cuatro melodías

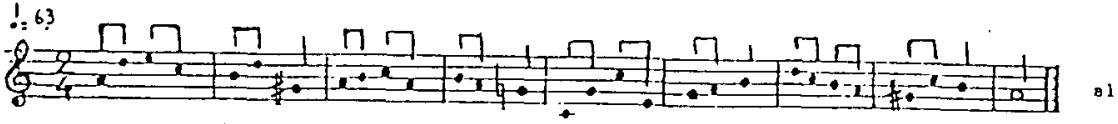

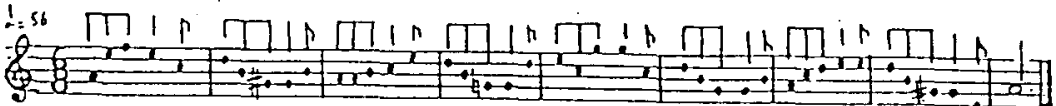

$\therefore 26$

$\therefore$ (2)

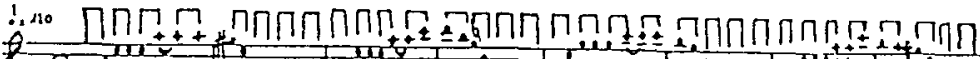

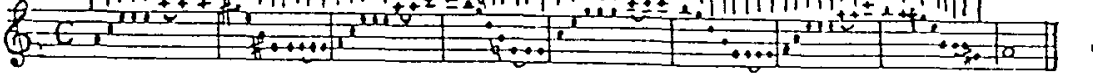

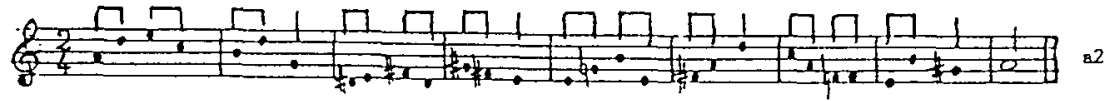

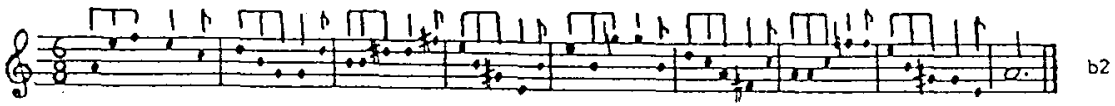

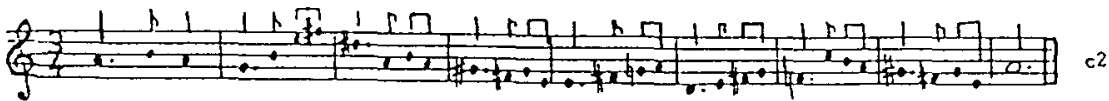

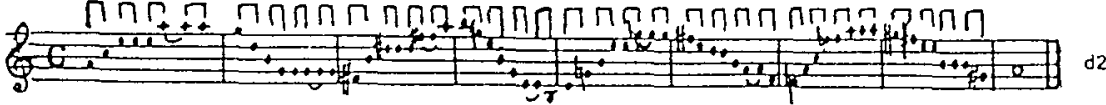

Figura 8

Estructuras prolongacionales de cada familia

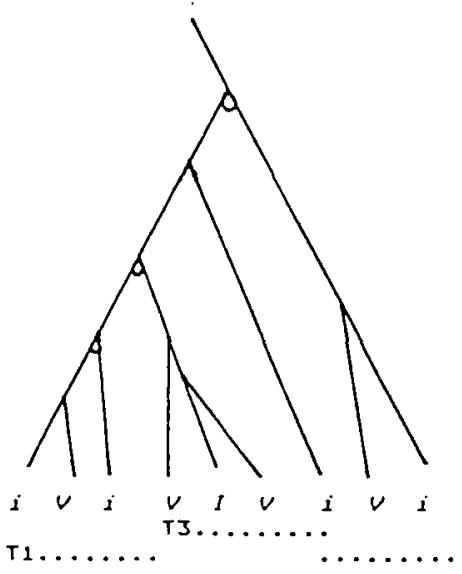

a) Esquemas de tensiones $y$ distensiones musicales en F1

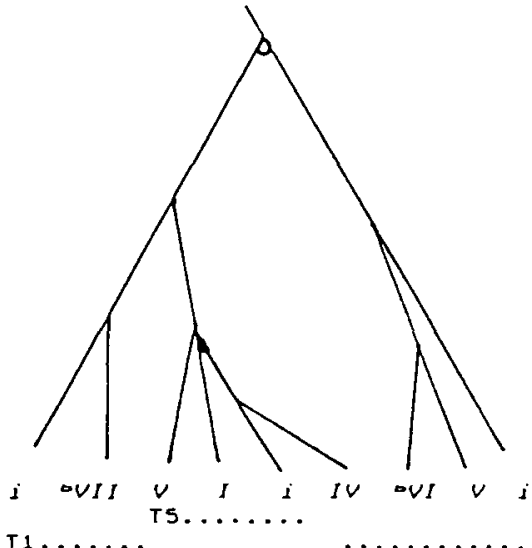

b) Esquemas de tensiones y distensiones musicales en $\mathrm{F} 2$ 
Cuando estas ocho melodías se interpretan con tonalidad diferentes, las estructuras subyacentes a cada familia se escriben según los grados de la armonía implícita. Esta notación representará la red jerárquica de tensiones y distensiones musicales sobre la que se desarrollan las melodías de cada familia, red que se puede representar en forma de gráfico (Fig. 8). La comparación de estos gráficos muestra que cada familia tiene una estructura prolongacional específica, que debería permitir establecer unas diferencias, a pesar de sus relaciones fisionómicas.

El experimento se desarrolló de la siguiente forma: en un primer grupo de sujetos se presenta dos veces seguidas una familia verdadera (a1, b1, c1, d1) (fase de adquisición). En el segundo grupo se presenta dos veces seguidas una familia falsa (a1, b2, c1, d2). En los dos casos a continuación se presentan las ocho familias mezcladas; la tarea consiste en identificar las melodías que se habían presentado inicialmente (tarea de reconocimiento). La hipótesis experimental es la siguiente: si las reglas de la reducción describen un fenómeno real, los sujetos del primer grupo deberían llegar a abstraer en la fase de adquisición la relación de parentesco característica de las 4 melodías presentadas, lo que inmediatamente les permitiría obtener unas puntuaciones de reconocimiento más altas que los del segundo grupo. La ausencia de diferencias significativas entre los dos grupos indicaría, por el contrario, que no se producía el fenómeno de abstracción de las organizaciones subyacentes. El experimento se realizó con 80 músicos y 80 sujetos que no lo eran, en dos situaciones experimentales: una en la que las melodías tenían la misma tonalidad, y otra en la que tenían tonalidades diferentes. Se vio muy claramente que las puntuaciones de identificación correcta eran significativamente más elevadas en los sujetos del primer grupo: por tanto, un oyente es capaz de abstraer la estructura subyacente común a 4 melodías (a1, b1, c1, d1) con aspectos superficiales muy distintos. Además puede diferenciar dos familias de melodías semejantes en función de su estructura subyacente. Esta diferencia de puntuación se observa en las dos situaciones experimentales, lo cual es un resultado fundamental: efectivamente, si el oyente es capaz de percibir la relación de parentesco entre dos melodías que no tienen las mismas notas, ni los mismos intervalos, ni los mismos ritmos, ni los mismo contornos melódicos, es que existe un nivel de codificación en el que la frase musical está representada por la red jerárquica de sus relaciones subyacentes.

Por último, estos resultados se observaron en músicos y no músicos. Sin duda la educación musical tiene una influencia significativa: las puntuaciones de los músicos eran sistemáticamente más altas. Sin embargo, la falta de interacción entre los diferentes factores manipulados en el experimento indica, de forma inequívoca, que los músicos no muestran una competencia que no existiría en los no músicos: las únicas diferencias se refieren a los resultados.

Este experimento confirma la validez psicológica de las hipótesis de reducción del modelo de Lerdahl y Jackendoff. El hecho de aparecer esos resultados con no músicos refuerza la idea de un oyente inteligente y activo, dotado de una competencia musical suficiente que le permite identificar el discurso musical tonal sin necesidad de un largo y tedioso aprendizaje previo. 


\section{CONCLUSION}

Es cierto que la generalización de este tipo de resultados ${ }^{6}$ podría tener importantes repercusiones no sólo en una enseñanza de la música destinada a un público no especialista, sino también, y fundamentalmente, en nuestra opinión, en la enseñanza dirigida a profesionales de la música. Efectivamente, nos parece interesante señalar que en varias de nuestras instituciones (CNR, ENM, Academia de formación profesional del oficio de músico de orquesta, etc.) se desarrolla una enseñanza basada, esencialmente, en los métodos de aprendizaje por condicionamiento operante: por ejemplo, la preparación de un concurso se resume, en la mayoría de los casos, a la interpretación una y otra vez, incansablemente, de la obra exigida por el programa, hasta el día J, como si de alguna forma su conocimiento y su dominio dependieran del número de repeticiones. No estaría de más preguntarse si este tipo de preparación no mata la dimensión creadora de la interpretación de la obra, que aparece como algo más cercano a una ejecución técnica, o mecánica, que a un verdadero acto de comprensión y comunicación musical?. En relación con esto, nos parece interesante constar que en otros países, como Inglaterra, Alemania o los Estados Unidos, país en que las teorías del musicólogo $\mathrm{H}$. Schenker constituyen el $\mathrm{ABC}$ de la enseñanza, los métodos pedagógicos son muy distintos. David Dolan ${ }^{8}$ nos comentaba que a todo instrumentista que está preparando un concurso se le invita a hacer un trabajo de improvisación clásica consistente en aislar los distintos niveles de estructuras subyacentes de la obra, y realizar una serie de variaciones sobre estas estructuras con el fin de asimilar mejor la especificidad y originalidad de la derivación final elegida por el compositor. Es evidente que este tipo de trabajo implica un conocimiento en profundidad no sólo de la obra y del lenguaje en el que está incluida, sino también de los procesos implicados en la creación musical. Ante este tipo de aproximación, nos parece que sería muy urgente que los profesores de música franceses reflexionaran seriamente sobre los modelos psicológicos implicados en su método de enseñanza, con el fin de desarrollar unos programas pedagógicos que hagan aflorar la competencia creadora de sus alumnos.

\section{Notas}

1 "El zorro devora al perro" y «el zorro es devorado por el perro".

2 Debemos señalar que esto podría darse también en muchos casos de comunicación verbal.

3 Sobre este punto remitimos al lector a unas interesantes reflexiones de B. Schloezer sobre las derivaciones de determinadas formas de cadencia en Bach (p. 124 y siguientes) y sobre las nociones de esquema formal y estructura orgánica en música.

${ }^{4}$ Para una revisión detallada de las tres categorías de factores ver Bigand, 1989.

5 Dos tónicas con diferente duración y estabilidad métrica, no tienen la misma importancia estructural.

6 Para profundizar en este planteamiento, revisar los interesantes resultados obtenidos por I. Deliège (1989-1990).

${ }^{7}$ No se trata de recriminar a los instrumentistas, que son los que más sufren con una enseñanza que realmente no quieren.

${ }^{8}$ Profesor de la City University de Londres, profesor de doctorado en el laboratorio de psicología de la cultura de la Universidad de París X. 


\section{Referencias}

Bakoni, M., Dalmonti:, R. y Jacobini, C. (1989). "Relations entre musique et poésie dans les airs de Giovanni Legrenzi». En S. McAdams, I. Deliège (ed.), La musique et les sciences cognitives, Bruselas, P. Mardaga, 46-74.

Bigand. E. (1989). Perception et comprébension des phrases musicales, tesis de doctorado en psicología, Universidad París X-nanterre (no publicado).

BIGAND. E. (1990). "Abstraction of Two Forms of Underlying Structure in a Tonal Melody", Psychology of Music, vol. 18, n. ${ }^{\circ} 1,45-60$.

Boul.tz, P. (1985). Quoi, quand, comment, la recherche musicale, París, Christian Bourgeois.

Chomsky, N. (1969). Le langage et la pensée, trad. Calvet, París, Payot.

Cı.лкке, E. (1989). "Considérations sur le langage et la musique", in S. McAdams, I. Deliège (ed.), La musique et les sciences cognitives, Bruselas, P. Mardaga, 24-42.

Di:I.II:GI: C. (1984). Les fondements de la musique tonale, París, J. C. Lattès.

Dit.ItGE, I. (1985). Les règles préférentielles de groupement dans la perception musicale, Tesis universitaria libre de Bruselas, Bélgica.

Di:1.IE:GE, I. (1987). «Grouping conditions in listening to music: an approach to Lerdahl Jackendoff's grouping preference rules", Music Perception, 4, (4), 325-360.

DE1.IEGE, I. (1989). «Approche perceptive des formes musicales contemporaines». En S. MacAdams, I. Deliège (ed.), La musique et les sciences cognitives, Bruselas, P. Mardaga, 305-326.

Dis.Is:GE. I. (1990). "Mecanisms of cues extraction in musical grouping: Study of Sequenza 6 by L. Berio", Psychology of music, vol. 18, 1, 18-44.

Francis. R. (1958). La perception de la musique, París, Vrin.

IMBi:RTY, M. (1979). Entendre la musique: semántique psychologique de la musique, tomo 1, París, Dunond.

IMiв:кт, M. (1981). Les écritures du temps: Sémantique psycbologique de la musique, tomo 2, París, Dunod.

IRCAM. (1989). Rapport annuel d'activités.

KRumhANSI., C. y Kessilik, E. (1982). "Tracing the dynamic changes in perceived tonal organisation in a spacial representation of musical keysé, Psychological Review, 89, 334-368.

LIRDAHI.. F. (1987). "Cognitive constraints on compositional systems». En J. Sloboda (ed.), Generative process in music, Oxford, Clarendo Press, 231-259.

Lı:RDAHı., F. (1989). “Structure de prolongation dans l'atonalité». En S. MacAdams, I. Deliège (ed.), La musique et les sciences cognitites, Bruselas, P. Mardaga, 102-135.

Lierdahl, F. y Jackendoff, R. (1983). A Generative theory of tonal music, Cambridge, MIT Press.

Lidov, D. (1975). "A Melody Writing Algorithm Using a Formal Language Model", trad. Latraverse F. \& Nattiez J. J. En On the Musical Phrase, Monographies de sémiologie et d'analyse musicale, Facultad de Música, Universidad de Montreal, 11-34.

MAssimo-Piattelli-Palmiekini. (1979). "A propos des programmes scientifiques et de leur noayau central». En Theories du langage et théories de l'apprentissage, París, Seuil, 19-47.

Mi:yik, L. (1973). Explaining music: Essays and explorations, Berkeley, California: University of California Press.

Moscato, M. y Wittwek. J. (1981). "La psychologie du langage», Que sais-je?, n. ${ }^{\circ}$ 1736, París, PUF.

NAi"Tll:\%. J.-J. (1987), Musicologie générale et sémiologie, París; Christian Bourgeois.

Nolz:1: G. (1980). De la perception à la comprébension du langage, París: PUF.

Paimik. C. y Krumhansi. C. (1987a). «Independant Temporal and Pitch Structures in Determination of Musical Phrases", Journal of Experimental Psychology: Human Perception and Performance, 13, (1), 116-126.

PAimik, C. y KRumhansl. C. (1987b). "Pitch and temporal contributions to musical phrase perception: Effects of harmony, performance timing, and familiarity", perception and psychophysics, $41,(6), 505-518$.

Rusi:n, C. (1978). Le style classique, Haydn, Mozart, Beethoven, trad. M. Vignal, París, Gallimard.

Ruwl:T, N. (1972). Language, musique et poésie, París: Seuil.

ScHI.ol:/: R. B. de. (1947). Introduction à J. -S. Bach, París: Gallimard.

SI:RAliNI:, M.-L. (1989). "The Cognitive Reality of Hierarchich Structure in Music", Music Perception, vol. 6, n. $4,397-430$.

SuNibis:RG. J. у LiN⿴囗i om. B. (1976). «Gneerative theories in language and in music descriptionsé, Cognition, 4, 99-122. 
Hacia una formalización de los procesos implicados en la comprensión musical. E. Bigand CLEE, 1991, 9, pp. 71-88

Datos sobre el autor: Doctor en Psicología y profesor en las Universidades de París-X (Psicología) y de Aix-Marselle-I (Musicologia).

Dirección: Universidad de París-X, Faculté de Psychologie, Paris.

Artículo orginal: Vers la formalisation des processus impliqués dans la compréhension musicale. Les Sciences de l'Education, 1990, 3-4, 85-108. Reproducido con autorización. Traducción de Inés Marichalar. $\odot$ de la traducción al catellano, CL\&E, 1991.

๑ de todos los artículos. Deberá solicitarse por escrito autorización de CL\&E para el uso en forma de facsímil, fotocopia o cualquier otro medio de reproducción. CL\&E se reserva el derecho de interponer las acciones legales necesarias en aquellos casos en que se contravenga la ley de derechos de autor. 